\title{
Thickening of the walls of deep brain abscesses is associated with macrophage infiltration
}

\author{
ZUOCHENG YANG ${ }^{1}$, YAKUN YANG $^{1}$, XUELING $^{2}{ }^{2}$, NING LIU $^{1}$, PENGFEI WANG $^{1}$, \\ LINPENG ZHANG $^{1}$, MINGYANG HAN ${ }^{1}$ and SONG HAN ${ }^{1}$
}

Departments of ${ }^{1}$ Neurosurgery and ${ }^{2}$ Pathology, Sanbo Brain Hospital, Capital Medical University, Beijing 100093, P.R. China

Received November 22, 2019; Accepted November 11, 2020

DOI: $10.3892 /$ etm.2021.10514

\begin{abstract}
The present study aimed to compare the thickness of brain abscesses in the deep and the superficial brain and to investigate the factors that influence the capsule of brain abscesses. The thickness of the brain abscess wall was evaluated on imaging. Bacteriological examination was performed on the abscess pus and wall, and immunohistochemical staining was used to count the number of macrophages. Kaplan-Meier curves were used to analyze overall survival. The results indicated that the wall of deep-brain abscesses was thicker than that of superficial abscesses. There was a difference in the extent of macrophage infiltration of deep- and superficial-brain abscess walls, and differences in the extent of macrophage infiltration in the wall of brain abscesses caused by various microorganisms were statistically significant. Of note, among the brain abscesses caused by Staphylococcus, the extent of macrophage/microglia infiltration and the thickness of the wall of the deep-brain abscesses were greater than those of superficial-brain abscesses and there was a positive correlation between the number of macrophages and the thickness of the abscess wall. The overall survival (OS) of patients with deep-brain abscess was not significantly shorter than that of patients with superficial-brain abscess. Furthermore, OS was not significantly different among groups of patients receiving different types of treatment. In conclusion, the wall of deep-brain abscesses is thicker than that of superficial abscesses and the infiltration of macrophages is abundant. The thick wall of abscesses in the deep brain may be associated with macrophage infiltration.
\end{abstract}

\section{Introduction}

Brain abscess is a serious and life-threatening infection, although advances in radiology, diagnostics and treatment have

Correspondence to: Dr Song Han, Department of Neurosurgery, Sanbo Brain Hospital, Capital Medical University, 50 Yi-Ke-Song Road, Beijing 100093, P.R. China

E-mail: hansong990913@ccmu.edu.cn

Key words: infection, abscess wall, deep brain, macrophage led to a reduction in associated mortality from 40 to $10 \%$ over the last 60 years (1). The diagnosis of brain abscess is most commonly validated by craniocerebral MRI, particularly using diffusion-weighted imaging (2) and the reported incidence of brain abscess ranges from 0.3 to 1.8 cases per 100,000 individuals (3-6). Although the overall incidence of brain abscess has declined, the incidence remains high in specific populations, including those with human immunodeficiency virus, a history of treatment with immunosuppressive drugs, disruption of the natural protective barriers surrounding the brain and those with a systemic source of infection (1,7-10). Most patients with brain abscess have predisposing factors (4,10-12). For instance, bacterial transmission may be caused by penetrating trauma, neurosurgery or otorhinolaryngological infection $(10,11)$.

A brain abscess is a focal suppurative process in the brain parenchyma. The first stage of brain-abscess formation may lead to perivascular inflammation (13). Subsequently, a capsule is formed, which evolves over approximately two weeks into a well-vascularized wall surrounding the necrosis $(9,14)$. A post-contrast MRI scan displays obvious ring-shaped enhancement of the abscess wall. While the formation of an abscess wall is important for limiting pathogen dissemination during the development of a brain abscess, the thickness of a brain abscess wall may increase the difficulty of stereotactic aspiration and also decrease the curative effect of antimicrobial therapy $(15,16)$. However, studies regarding the wall of brain abscesses are currently limited.

The formation of a brain-abscess wall refers to the formation of a fibrotic wall, and the extensive proliferation and activation of fibroblasts $(17,18)$. Activated macrophages regulate fibrogenesis by providing cytokines and growth factors that modulate the proliferation and collagen synthesis of fibroblasts (19). Research on fibrotic walls in the central nervous system (CNS) has identified that alternatively activated M2 macrophages and microglia accumulate during the course of brain-abscess development (20).

The distinction between deep and superficial abscesses is not clearly defined. In the present study, abscesses of the basal ganglia region and thalamus were defined as deep abscesses according to the division of midline gliomas (21).

The aim of the present study was to explore differences in the thickness of deep and superficial brain abscesses and investigate the factors that affect the capsule of brain abscesses. 


\section{Materials and methods}

Patients. Information on patients with brain abscess treated at Sanbo Brain Hospital, Capital Medical University (Beijing, China) between December 2008 and December 2018 was retrospectively reviewed. Inclusion criteria for this group were adult and pediatric patients diagnosed with a brain abscess and admitted to the above hospital. The diagnosis of brain abscess was mainly based on symptomatology, laboratory examination and imaging findings $(2,11)$. In total, 95 patients with complete imaging information were selected for inclusion in the study. Unfortunately, due to the limited samples for immunohistochemical examination for macrophages, only 37 patients underwent surgical resection and had sufficient tissue specimens for relevant tests. The study cohort was divided into those with deep abscesses (thalamus and basal ganglia region) and superficial abscesses (frontal lobe, parietal lobe, temporal lobe, occipital lobe and cerebellum).

The enrolled cases were diagnosed by $\mathrm{CT}$ and/or MRI and pathology. Age, sex, clinical presentation, duration of symptoms/signs in patients and localization of the abscess were identified.

Measurements of the thickness of the brain abscess wall. All enrolled patients underwent post-contrast MRI and/or CT scanning prior to treatment. The abscess wall thickness was measured using miPlatform Viewer version 2.0 (Hinacom Software and Technology, Ltd.). The thickness of the abscess wall was measured at the thickest and thinnest places in different axial slices ( $\geq 3$ slices; layer thickness, $5 \mathrm{~mm}$ ). The median thickness in each layer was calculated and the mean values of the medians were defined as the thickness of the abscess wall.

Culture and identification of pathogenic microorganisms. According to our laboratory's standard operation procedure, all samples of the pus or wall of brain abscesses were sent to the microbiology laboratory at Sanbo Brain Hospital, Capital Medical University (Beijing, China) for urgent microscopy, culture and sensitivity testing. The pus and wall of brain abscesses were collected in a sterilized vial and snap-frozen in liquid nitrogen immediately after surgery/aspiration.

Immunohistochemistry. The group of patients with abscesses included 10 cases with abscess in the deep brain and 27 in the superficial brain. The samples were fixed with $10 \%$ neutral buffered formalin and further embedded in paraffin. Antigen retrieval was facilitated by heat and endogenous peroxidases were neutralized with $3 \%$ hydrogen peroxide in a routine fashion. Primary antibodies against CD68 (anti-CD68 antibody; 1:100; cat. no. ab125212; Abcam) were applied overnight at $4{ }^{\circ} \mathrm{C}$. According to the manufacturer's protocol, the Poly-HRP Anti-Mouse/Rabbit IgG Detection System (cat. no. PV-9000; Zhongshan Goldenbridge-Bio) was employed with incubation for $30 \mathrm{~min}$ at $37^{\circ} \mathrm{C}$. The cutoff value was $10 \%$ for CD68. This approach was identical to that used in a previous study by our group (22).

The samples were examined using a Nanozoomer Digital Pathology (NDP; Hamamatsu Photonics; magnification, x100), which was used to identify five regions (hot spots) with the highest density of stained $\mathrm{CD}^{+} 8^{+}$macrophages or microglia. The number of stained macrophages or microglia was counted in every area at a higher magnification (magnification, $\mathrm{x} 400$ ). The average of the three highest values was used as the final value $(23,24)$.

Statistical analysis. Statistical analyses were performed using the statistical analysis software package SPSS (version 25.0; IBM Corp.). Values are expressed as the mean \pm standard errors or $\mathrm{n}(\%)$. Unpaired student's t-test and Mann-Whitney U test were utilized. The thickness of the brain-abscess wall and the extent of macrophage infiltration among different pathogenic microorganisms were determined using ANOVA. Scheffe's test was used for post-hoc multiple-comparison testing, with a Scheffe-corrected alpha of 0.05 considered to indicate statistical significance. Correlations were assessed using Spearman test. Overall survival (OS) was measured from the date of treatment until death or the date of the last follow-up. Survival analysis results were performed using Kaplan-Meier (K-M) survival curves with log rank testing. $\mathrm{P}<0.05$ was considered to indicate statistical significance.

\section{Results}

Patients. All patients were diagnosed with a brain abscess between December 2008 and December 2018. The mean age of the participants was 41.29 years (range, 5-71 years) and the male-to-female ratio was 1:0.44 (Table I).

Clinical findings. In patients with deep-brain abscess, the prevalent symptoms were impaired consciousness and focal neurological deficits. However, these symptoms were less common in patients with superficial-brain abscess, who were more likely to experience symptoms such as nausea, vomiting and fever (Table I).

Thickness of the abscess wall and macrophages in brain abscesses. In total, 95 patients with brain abscess were reviewed. The location of the brain abscesses was as follows: A total of 22 patients had deep brain abscess (thalamus and basal ganglia region) and 73 patients had superficial brain abscess (frontal lobe, parietal lobe, temporal lobe, occipital lobe and cerebellum). According to the independent-samples t-test, the thickness of the wall of the brain abscesses was significantly different between the deep $(4.65 \pm 2.66 \mathrm{~mm})$ and the superficial $(3.16 \pm 1.92 \mathrm{~mm}$ ) groups (95\% CI: 0.48-2.51; $\mathrm{P}=0.004$; Fig. 1 and Table II). To study the infiltration of macrophages or microglia in the brain abscesses, CD68-stained slides were collected from 37 patients and the number of macrophages/microglia was counted. This group included 10 patients with deep-brain abscess and 27 patients with superficial-brain abscess. There was a significant difference between the number of macrophages/microglia in the deep-brain abscess $(41.40 \pm 11.44)$ and the superficial-brain abscess walls $(19.67 \pm 15.77$; $95 \% \mathrm{CI}$ : 0.63-22.84; P=0.04; Fig. 2 and Table II). However, there was no statistically significant linear correlation $(\mathrm{P}=0.99$; Table II) between the thickness of the brain abscess wall and the number of macrophage infiltrations, as calculated with a bivariate correlation. 
Table I. Characteristics of the patients $(n=95)$.

\begin{tabular}{|c|c|}
\hline Characteristic & Value \\
\hline \multicolumn{2}{|l|}{ Sex } \\
\hline Male & $66(69.47)$ \\
\hline Female & $29(30.52)$ \\
\hline Age (years) & $41.29 \pm 16.76(5-71)$ \\
\hline \multicolumn{2}{|l|}{ Region of abscess } \\
\hline $\begin{array}{l}\text { Midline deep brain (thalamus and } \\
\text { basal ganglia region) }\end{array}$ & $22(30.14)$ \\
\hline $\begin{array}{l}\text { Shallow brain (frontal lobe, parietal } \\
\text { lobe, temporal lobe, occipital lobe } \\
\text { and cerebellum) }\end{array}$ & $73(76.84)$ \\
\hline \multicolumn{2}{|l|}{ Signs/symptoms in the deep brain } \\
\hline Focal neurological deficits & $16(72.73)$ \\
\hline Fever & $14(63.64)$ \\
\hline Headache & $7(31.82)$ \\
\hline Nausea/vomiting & $5(22.73)$ \\
\hline Impaired consciousness & $5(22.73)$ \\
\hline Seizures & $2(9.09)$ \\
\hline \multicolumn{2}{|l|}{ Signs/symptoms in the shallow regions } \\
\hline Headache & $40(54.79)$ \\
\hline Focal neurological deficits & $30(41.10)$ \\
\hline Fever & $20(27.40)$ \\
\hline Headache & $40(54.79)$ \\
\hline Nausea/vomiting & $16(21.92)$ \\
\hline Seizures & $12(20.27)$ \\
\hline Impaired consciousness & $7(9.89)$ \\
\hline \multicolumn{2}{|l|}{ Predisposing conditions in the deep brain } \\
\hline Diabetes & $4(18.18)$ \\
\hline Surgery & $5(22.73)$ \\
\hline None & $13(59.09)$ \\
\hline \multirow{2}{*}{\multicolumn{2}{|c|}{$\begin{array}{l}\text { Predisposing conditions in the shallow } \\
\text { regions }\end{array}$}} \\
\hline & \\
\hline Surgery & $19(26.03)$ \\
\hline Congenital heart disease & $6(8.22)$ \\
\hline Intracranial tumors & $5(6.85)$ \\
\hline Cerebral hemorrhage & $4(5.48)$ \\
\hline Diabetes & $3(4.11)$ \\
\hline Injury & $3(4.11)$ \\
\hline Leukemia/bone-marrow transplantation & $2(2.74)$ \\
\hline None & $31(42.47)$ \\
\hline \multicolumn{2}{|l|}{ Sequelae in the deep brain } \\
\hline Limb dyskinesia & $5(22.73)$ \\
\hline Seizures & $1(4.55)$ \\
\hline None & $16(72.7)$ \\
\hline \multicolumn{2}{|l|}{ Sequelae in the shallow brain } \\
\hline Limb dyskinesia & $6(8.22)$ \\
\hline Seizures & $5(6.85)$ \\
\hline Headache & $3(41.10)$ \\
\hline Hypaesthesia & $1(1.37)$ \\
\hline None & $58(79.45)$ \\
\hline
\end{tabular}

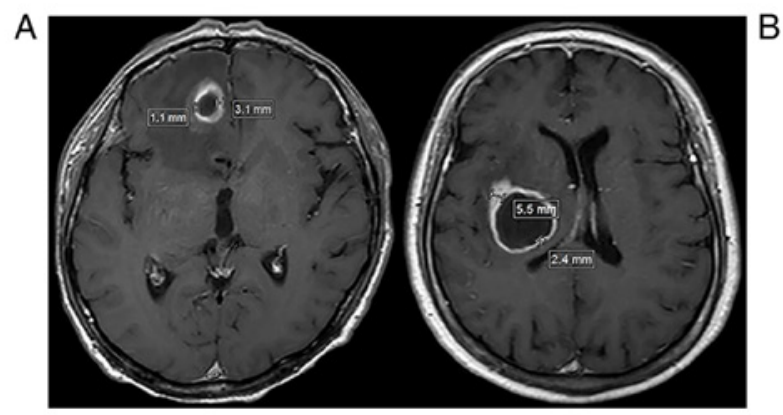

Figure 1. Thickness of the wall of deep-brain abscess is higher than that of superficial-brain abscess. (A) Thicknesses of the abscess wall in right frontal lobe (superficial brain) was measured on enhanced T1 MRI. (B) Thicknesses of abscess wall in the left basal ganglia (deep brain) was measured on enhanced T1 MRI.

Duration of the symptoms or signs. The different symptoms or signs between the two types of brain abscesses were present for $41.88 \pm 90.15$ days (ranging from 1 to 730 days; median, 15 days). The median duration of symptoms for patients with deep-brain abscess $(23.58 \pm 30.63$ days; median, 15 days) was shorter than for those with superficial abscess (47.00 \pm 90.41 days; median, 14 days), but there was no statistically significant difference according to nonparametric testing ( $\mathrm{P}=0.654$; Table II); furthermore, there was no correlation between the thickness of the brain abscess wall and symptom duration $(\mathrm{P}=0.21$; Table II) according to bivariate correlation analysis.

Macrophages and microorganisms. Taken from the pus and wall of the abscess, abscess cultures were positive in 34 of 71 patients who received surgery or drainage of the abscess (Table III). The most common species were Gram-positive bacteria, followed by Gram-negative bacteria and fungi (Table III). The thickness of the abscess wall exhibited no significant difference between different underlying organisms according to one-way ANOVA $(\mathrm{F}=2.00, \mathrm{P}=0.24$; Fig. 3).

Among the 37 patients with CD68-stained slides, 16 were positive on brain-abscess pus and/or wall bacterial culture (Table IV). Of note, according to one-way ANOVA and Scheffe's post-hoc tests, the extent of macrophage infiltration in brain abscesses (Gram staining-positive group: $n_{1}=35.71 \pm 12.51$ vs. Gram staining-negative group: $\mathrm{n}_{2}=58.75 \pm 16.15$ vs. fungal group: $\mathrm{n}_{3}=17.67 \pm 17.20$ vs. mixed bacterial group: $\left.\mathrm{n}_{4}=28.00 \pm 5.67\right)$ caused by various microorganisms was significantly different ( $\mathrm{F}=5.43, \mathrm{P}=0.014$; Fig. 4) and there was a significant difference between the gram-negative bacteria group and the fungi group in macrophage distribution according to Scheffe's post-hoc test $(\mathrm{P}=0.019)$. Among the cases of brain abscess caused by Staphylococcus, two patients had deep-brain abscesses and three had superficial-brain abscesses; the extent of macrophage/microglia infiltration in deep-brain abscesses $(47.00 \pm 11.97)$ was greater than that in the superficial-brain abscesses $(33.33 \pm 3.06, \mathrm{P}=0.03$; Table V). In addition, the thickness of the brain-abscess wall was significantly different between the deep $(5.60 \pm 1.98)$ and the superficial $(1.95 \pm 0.76)$ abscesses $(\mathrm{P}=0.04$; Table V). The correlation coefficient between the thickness of an abscess 
Table II. Summary of statistical results on the related factors in 95 patients.

\begin{tabular}{lcc}
\hline Index & $\begin{array}{c}\text { Statistical } \\
\text { method }\end{array}$ & P-value \\
\hline Thickness of abscess wall & $\mathrm{t}$-test & 0.004 \\
Deep-brain (mm) & $4.65 \pm 2.66$ & \\
$\quad$ Superficial brain (mm) & $3.16 \pm 1.92$ & \\
Number of macrophages/microglia & $\mathrm{t}$-test & 0.04 \\
$\quad$ Deep brain(n) & $41.40 \pm 11.44$ & \\
$\quad$ Superficial brain(n) & $19.67 \pm 15.77$ & \\
Thickness of abscess wall & Spearman test & 0.99 \\
Number of macrophages/microglia & & \\
Duration of symptoms & Mann-Whitney & 0.65 \\
$\quad$ Deep brain(day) & 15 & \\
$\quad$ Superficial brain(day) & 14 & \\
Thickness of abscess wall & Spearman test & 0.21 \\
Duration of symptoms & & \\
\hline
\end{tabular}

wall and the infiltrating number of macrophages was 0.09 $(\mathrm{P}=0.04$; Table $\mathrm{V})$, indicating a positive correlation.

Survival analysis. Follow-up was performed for 67 patients. Of these, 32 patients received surgery, 20 patients received puncture drainage and 15 patients were treated with drug therapy. The follow-up time ranged from 0.7-106 months, with a median follow-up time of 25.00 months. No recurrence of the brain abscesses was detected during follow-up. The OS of patients with deep-brain abscess was not significantly reduced compared with that of patients with superficial-brain abscess $(\mathrm{P}=0.31$; Fig. 5). Furthermore, there was no significant difference in OS among the different treatment groups ( $\mathrm{P}=0.74$; Fig. 6).

\section{Discussion}

In the present study, it was demonstrated that brain abscesses were different between the deep and superficial regions in terms of thickness of the abscess wall and infiltration by macrophages/microglia. Furthermore, the factors affecting the wall of a brain abscess were analyzed. The formation of a capsule in a brain abscess has a significant effect on limiting pathogen diffusion. Despite previous studies illustrating the mechanisms of fibrotic processes (25) in the CNS, these mechanisms remain to be fully elucidated and previous studies have rarely focused on the relationship between the location and the fibrosis of brain abscesses.

In the cohort of the present study, patients with abscess in deep regions more frequently had focal neurological deficits (72.73\%) and impaired consciousness (22.73\%) compared with patients with abscess in superficial brain regions (41.10 and 9.89\%, respectively). Furthermore, the complications of deep-brain abscess included dyskinesia following treatment, which caused most patients with deep-brain abscess to suffer with movement disorders during the follow-up (26).

By measuring the thickness of the wall of brain abscesses in images, it was determined that the wall of deep-brain abscesses was thicker than that of superficial-brain abscesses. The process of brain-abscess encapsulation involves the formation of a fibrotic wall. A key feature of fibrotic wall formation is extensive proliferation and activation of fibrotic tissue $(20,27,28)$; fibroblasts secrete $\alpha$-smooth muscle actin, which is a constituent of the fibrotic abscess capsule (29). Previous studies demonstrated that alternatively activated macrophages enhanced fibrogenesis of fibroblasts by providing profibrogenic factors, such as transforming growth factor $\beta 1$ and platelet-derived growth factors (19). Fibrotic wall formation was associated with increased numbers of alternatively activated microglia and macrophages (19) and relative markers for these cells were also indicated to increase, in accordance with fibrotic wall formation (20).

Microglia/macrophage activation is an important feature of a brain abscess (30). Studies have indicated that activated glial cells and infiltrated peripheral immune cells continuously release pro-inflammatory mediators. These mediators enhance the subsequent recruitment and activation of newly recruited inflammatory cells and glial cells; this effectively maintains the antimicrobial inflammatory response and ultimately achieves the goal of eliminating pathogenic microorganisms $(31,32)$. Of note, the present study indicated that the number of infiltrating macrophages in deep-brain abscesses was greater than that in superficial-brain abscess walls. Studies in the rat brain have indicated that macrophages are mainly distributed in the gray matter of the brain, particularly the basal ganglia, hippocampus, olfactory telencephalon and substantia nigra (33). In addition, the basal ganglia are rich in blood vessels, so the content of macrophages in brain abscesses of the basal ganglia may be higher.

There is a large time range in the symptom/sign duration, as was particularly evident in the case of a five-year-old female patient with epilepsy for $\sim 730$ days, who, due to poor economic conditions and personal factors, was not diagnosed accurately and treated effectively until they received surgical treatment at our hospital. At the final follow-up, it was determined that the seizure frequency had gradually decreased after the brain abscess was cured. The thickness of the wall of the brain abscess may change during a long evolutionary period. Comparing the CT appearances and the neuropathologic findings in dogs, Britt et al (13) and Enzmann et al (34) determined that ring-shaped contrast enhancement appeared on CT imaging in the early cerebritis stage and well-encapsulated brain abscesses occurred at the age of 14 days and beyond through the accumulation of fibroblasts and neovascularization, with the thickness and diameter of ring-like abscesses decreasing as cerebritis later receded. Pathological abscess formation processes may slow down in the chronically immunosuppressed host (35). However, the results of the present study indicated that time was not significantly correlated with the thickness of a brain abscess. There was no significant difference between the deep and superficial brain abscesses over time. This may be due to a number of reasons, including the fact that most patients present at the stage of capsule formation or later; the thickness of the wall of a brain abscess may undergo no significant changes in the absence of any therapeutic intervention. Similarly, pathophysiological analysis of brain abscesses suggests that activation of microglia/macrophages is also evident during early encephalitis (days 1-3) 

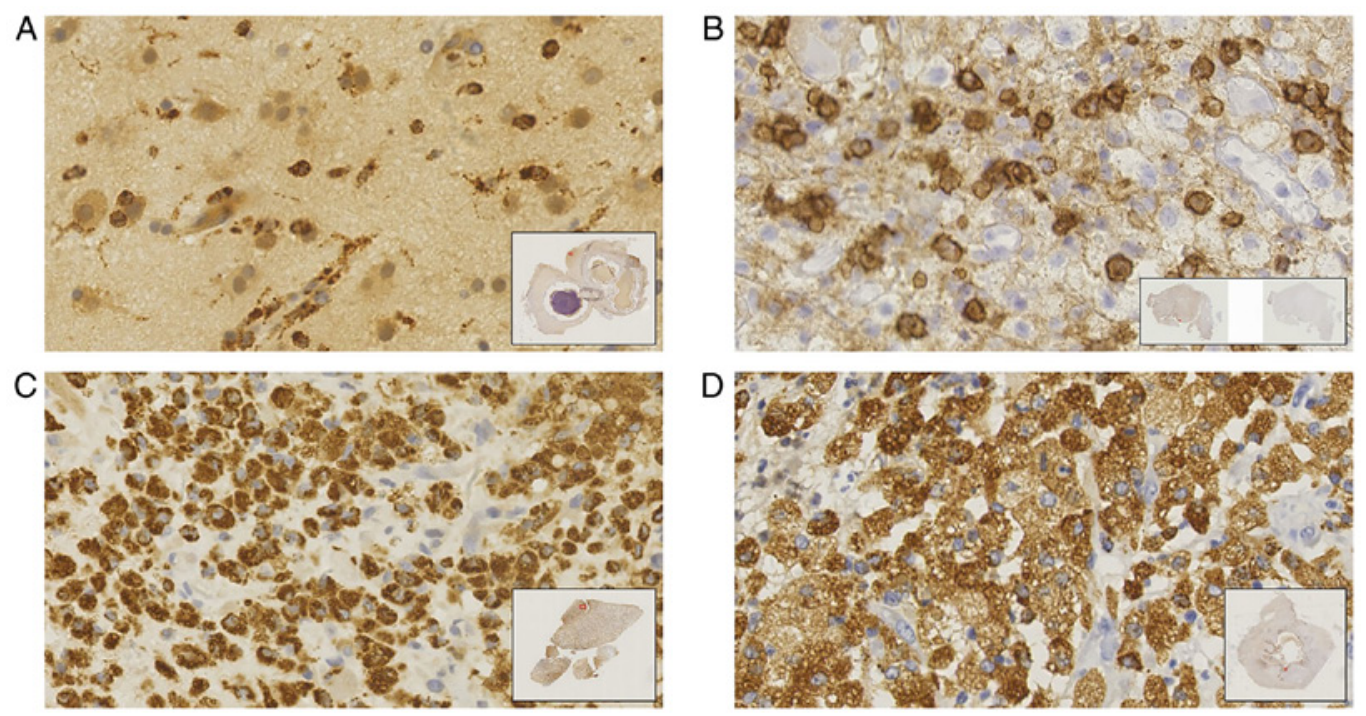

Figure 2. Macrophage/microglial infiltration in the brain abscess wall. (A and B) The number of macrophages/microglials in superficial-brain abscess walls $\left(\mathrm{n}_{\mathrm{A}}=18\right.$ and $\left.\mathrm{n}_{\mathrm{B}}=27\right)$ in the CD68 labeling. $(\mathrm{C}$ and $\mathrm{D})$ The number of macrophages/microglials in deep-brain abscess walls $\left(\mathrm{n}_{\mathrm{C}}=48\right.$ and $\left.\mathrm{n}_{\mathrm{B}}=47\right)$ in the $C D 68$ labeling. The small windows in the lower right corner of (A-D) images show the corresponding whole tissue slices, and the red box is the position corresponding to 400 times of the microscopical snapshot. $(A-D)=($ magnification, $x 400)$.

and continues throughout abscess development. The stage of advanced encephalitis (days 4-9) is associated with macrophage and lymphocyte infiltration; the final capsule phase occurs after day 10 and is associated with the formation of well-vascularized abscess walls (32). Combined with the results of the above study, the present study observed that the subsequent immune response to pathogenic bacteria became gradually stronger. The immune response to pathogens may be able to destroy surrounding normal brain tissue $(31,36,37)$. Thus, it is not surprising that mechanisms of reducing defensive responses and promoting wound repair and healing are part of immunity. Previous studies have demonstrated the mechanisms by which the innate immune response may be reduced, including the following: Reducing pathogen load, decomposing pro-inflammatory mediators, managing the negative feedback of activating immune cells and controlling the activation pathway (38). Van Rossum et al (39) indicated that peripheral macrophages and dead or dying immune cells spread throughout the vascular system and are eventually removed from circulation, although the movement of cells and solutes from the blood vessels to the brain parenchyma remains to be characterized (38). Due to the long duration of symptoms/signs (41.88 \pm 90.15 days) and differences in individual immunity and fibrosis, the imaging results of brain abscesses and the low bacterial culture rate of patients in this group indicate that most patients may be in the period of declining immune defense response. Macrophages/microglia were in retreat and the number of infiltrating macrophages in brain abscess was not linearly related to the thickness of the abscess wall. In addition, the time of symptoms/signs may not accurately reflect the pathophysiological stage of the brain abscess. It is possible that there is no linear correlation between the time of symptoms/signs.

The brain is well protected from invading pathogens by the two major barriers [the blood-brain barrier (BBB) and the blood-cerebrospinal fluid barrier]. However, bacteria have developed a wide variety of different strategies to cross these barriers and reach the CNS $(40,41)$. They use a variety of different virulence factors to invade the host cell, including factors that mediate intracellular survival, induction of host-cell signaling and inflammatory response; these factors enable them to attach to and traverse the two barriers (40). Certain mechanisms may vary with different pathogens (40). For instance, Streptococcus pneumoniae may translocate across the BBB by altering expression of the Neisseria adhesin A for attachment and interacting with the BBB (42-44). However, Escherichia coli relies on type 1 fimbriae, outer membrane protein A, IbeA; a virulence factor of Eschericia coli, and cytotoxic necrotizing factor 1 to attach and invade the BBB (45-48). Due to the small number of positive cases determined by bacterial culture in the present study, a detailed classification was not easy to perform using statistical methods and the following groups were created: The Gram staining positive group, Gram staining negative group, a fungal group and a mixed bacterial group. Different pathogenic bacteria of brain abscesses may have different effects on macrophage infiltration, but no studies have been performed to investigate such differences. In the present study, the extent of macrophage infiltration in fungal brain abscesses was the lowest, which may be related to their ability to evade an immune response by associating with Toll-like receptor (TLR) action and anti-inflammatory response (49); an example is C. albicans, which may induce immune suppression by activating TLR-2, leading to the release of IL-10, an anti-inflammatory cytokine that activates $\mathrm{CD} 4{ }^{+} \mathrm{CD} 25^{+} \mathrm{T}$-regulatory cells (50).

In the present study, the extent of macrophage infiltration caused by gram-negative bacteria in brain abscesses was the highest. However, the number of cases with a positive culture of gram-negative bacteria was lower in deep-brain abscesses and it was not possible to perform a statistical analysis. Therefore, a statistical analysis of relevant factors in brain abscesses caused by Staphylococcus, which is a common pathogenic bacteria in brain abscesses, was performed $(3,51)$. Of note, in the brain abscesses caused by 
Table III. Culture of pathogens isolated from 34 patients with brain abscess.

\begin{tabular}{lc}
\hline Organism & Number of patients \\
\hline Gram-positive bacteria & 18 \\
Streptococcus species & 6 \\
Staphylococci & 7 \\
Corynebacterium urealyticum & 1 \\
Gemella haemolysans & 1 \\
Other & 1 \\
Gram-negative bacteria & 10 \\
Pseudomonas aeruginosa & 5 \\
Proteus mirabilis & 1 \\
Klebsiella pneumoniae & 1 \\
Serratia glutinosa & 1 \\
Mixed bacterial pathogens & a \\
Fungi & 2 \\
Negative culture & 4 \\
\hline
\end{tabular}

${ }^{a}$ Mixed bacterial pathogens included Gram-positive and Gram-negative bacteria but the details of those species were unclear.

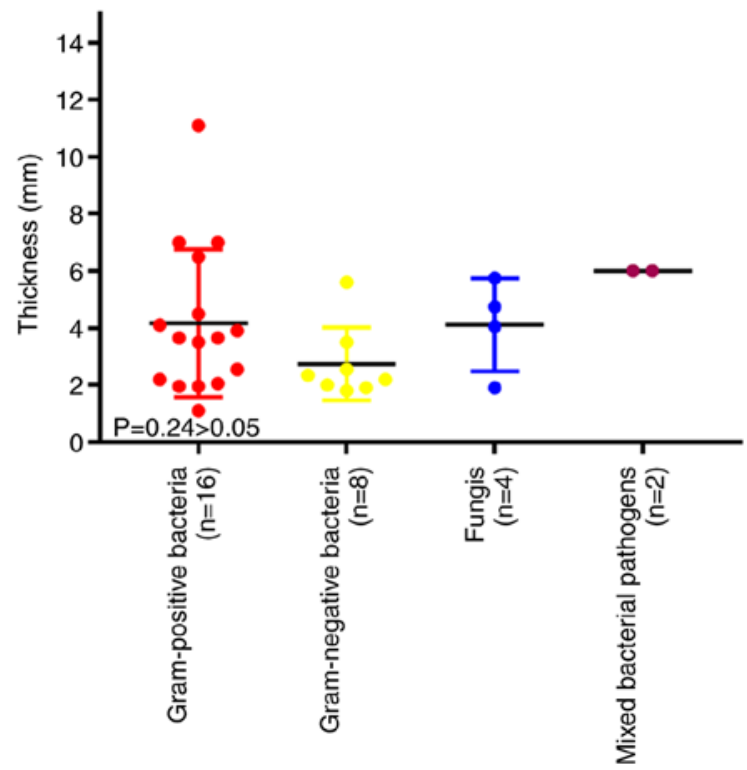

Figure 3. Mean thicknesses (mm) of brain abscess wall in all cases compared for various pathogens.

Staphylococci, the thickness of the deep-brain abscess wall was thicker than that of the superficial-brain abscess wall. The amount of macrophage infiltration in the deep brain abscess was higher than that in the superficial brain abscess and the thickness of the wall of brain abscesses was positively correlated with the number of macrophages. Certain models have demonstrated that Staphylococcus aureus and its cell wall product peptidoglycan had a strong stimulatory effect on pro-inflammatory mediators produced by primary microglial cells (36), inducing a rapid elevation and sustained high levels of pro-inflammatory cytokines and chemokines,
Table IV. The number of macrophages in 16 cases with positive bacterial culture was observed.

\begin{tabular}{lc}
\hline Organism & Number of patients \\
\hline Gram-positive bacteria & 7 \\
Staphylococci & 5 \\
Streptococcus species & 1 \\
Other & 1 \\
Gram-negative bacteria & 4 \\
Pseudomonas aeruginosa & 1 \\
Klebsiella pneumoniae & 1 \\
Serratia glutinosa & 1 \\
Proteus mirabilis & 1 \\
Mixed bacterial pathogens & a \\
Fungi $_{\text {Negative culture }}$ & 2 \\
\end{tabular}

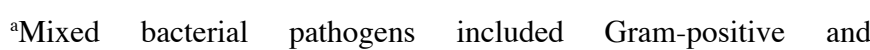
Gram-negative bacteria but the details of those species were unclear.

Table V. A summary of results on the thickness of abscess wall and macrophages/microglia in 5 patients with brain abscess caused by Staphylococcus.

\begin{tabular}{lcc}
\hline Index & $\begin{array}{c}\text { Statistical } \\
\text { method }\end{array}$ & P-value \\
\hline Thickness of abscess wall & t-test & 0.04 \\
Deep-brain (mm) & $5.60 \pm 1.98$ & \\
Superficial brain (mm) & $1.95 \pm 0.76$ & \\
Number of macrophages/microglia & t-test & 0.03 \\
Deep brain (n) & $47.00 \pm 11.97$ & \\
Superficial brain (n) & $33.33 \pm 3.06$ & \\
Thickness of abscess wall & Spearman test & 0.04 \\
Number of macrophages/microglia & $\mathrm{B}=0.09$ & \\
\hline
\end{tabular}

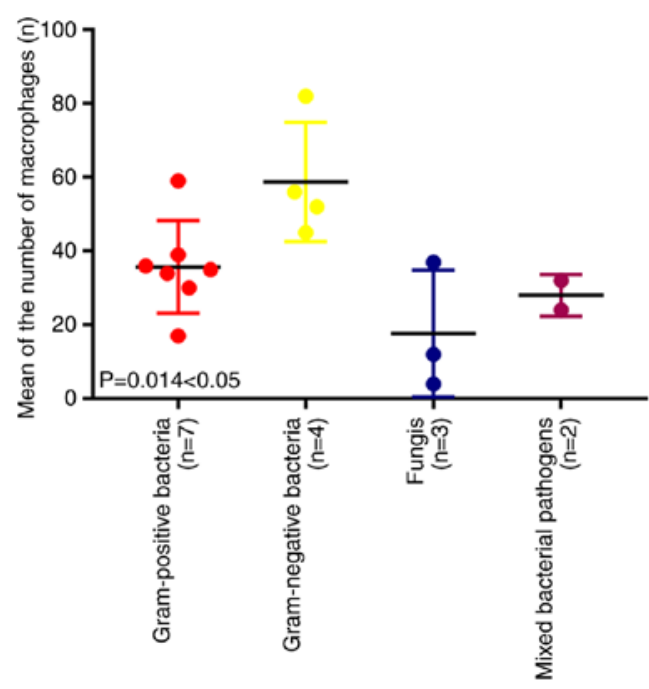

Figure 4. Mean number of macrophages/microglials (n) infiltrating in all brain abscess walls in various pathogens. 


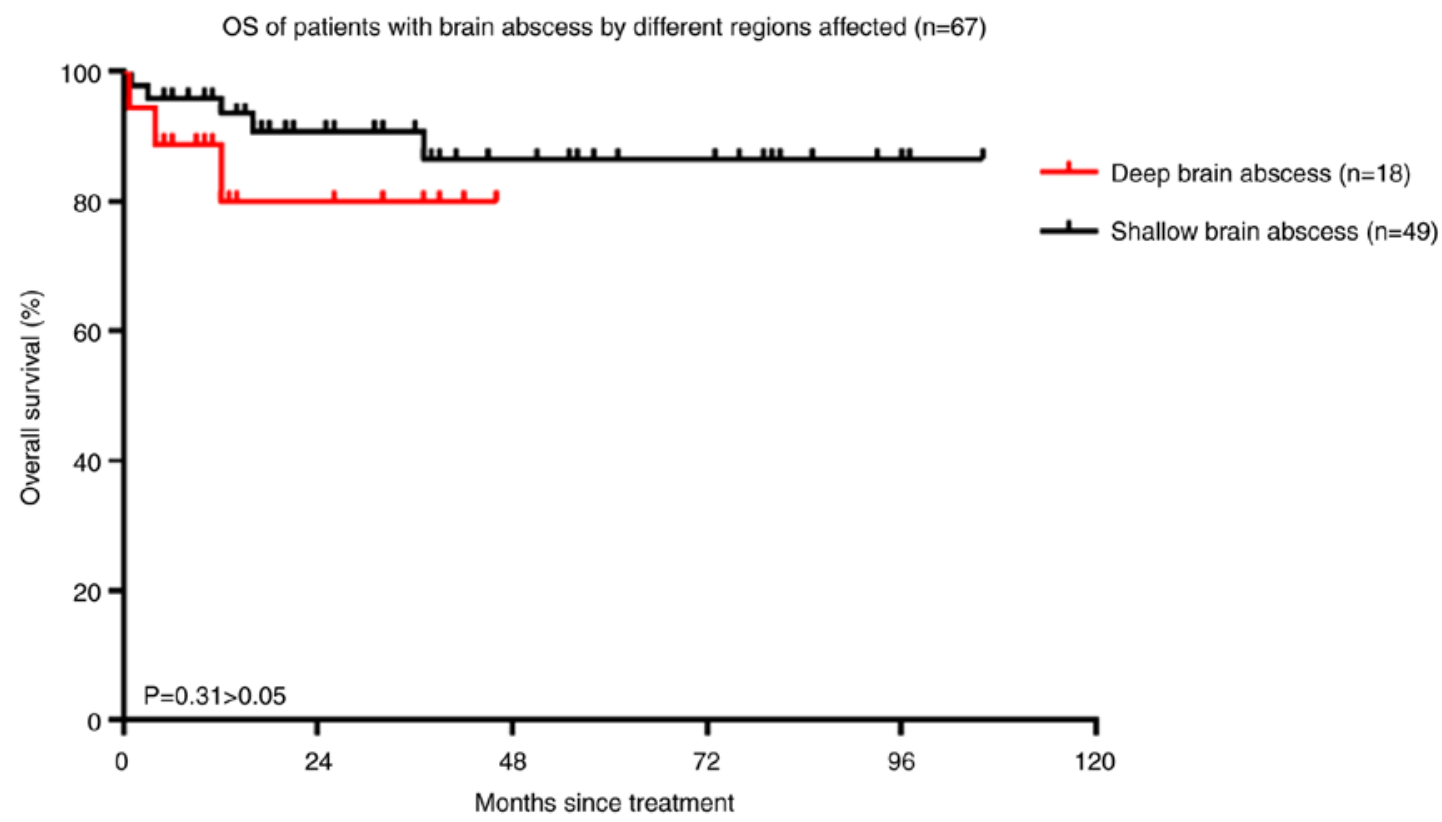

Figure 5. OS of patients based on brain abscess location. Patients with brain abscesses in the deep brain did not have a significantly better OS rate than patients with brain abscesses in the shallow brain $(\mathrm{P}=0.31)$. OS, overall survival.

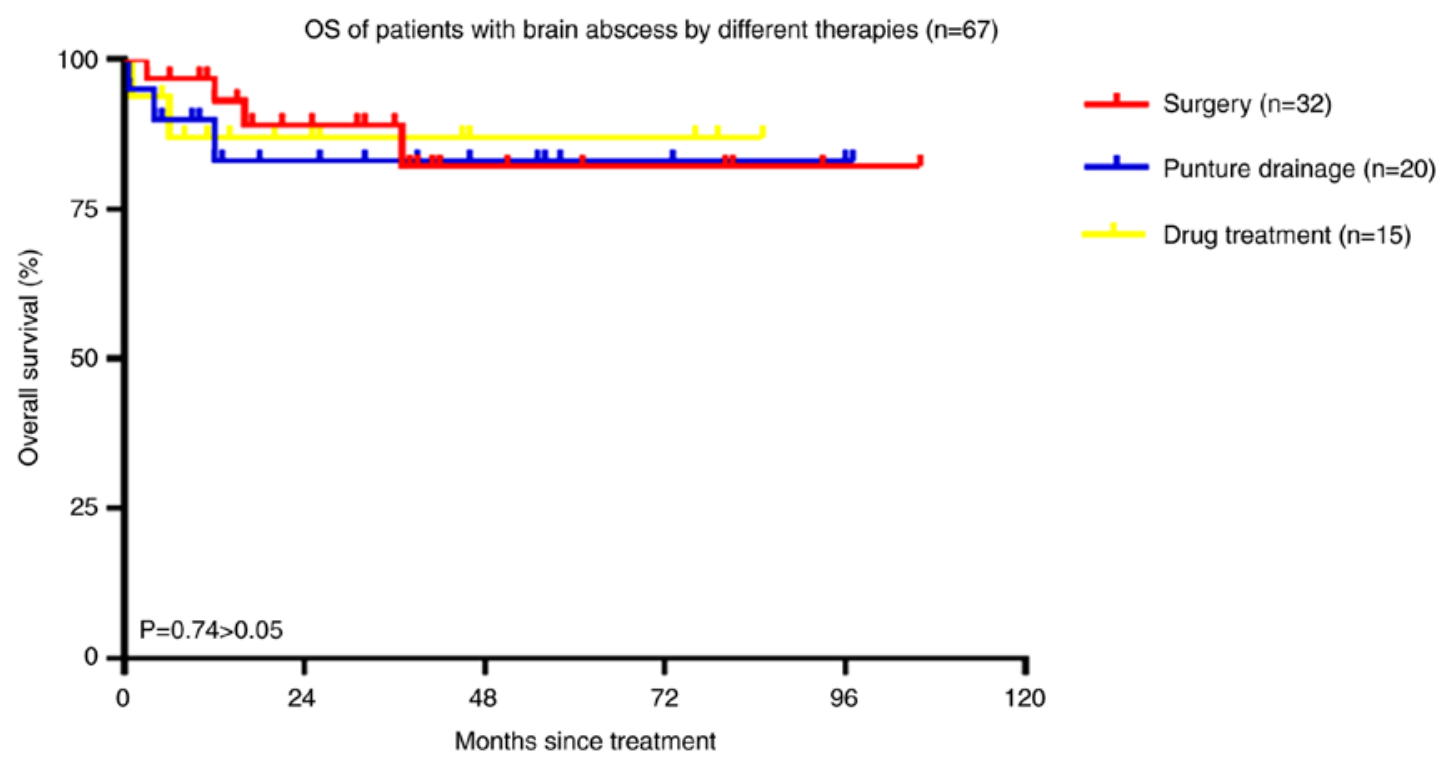

Figure 6. OS of patients based on therapies. Patients with brain abscesses who received surgery, puncture drainage and drug treatment exhibited no significant difference in OS $(\mathrm{P}=0.74)$. OS, overall survival.

including IL-1 $\beta$, TNF, IL-12 p40, C-X-C motif chemokine ligand 2, C-C motif chemokine ligand (CCL)2, CCL3 and CCL4 (52-54). The release of pro-inflammatory cytokines led to the destruction of the BBB and vascular cell adhesion molecules also promoted the involvement of macrophages in the evolution of brain abscesses (32). These changes are associated with the persistence of microglia/macrophages in brain abscesses. In brain abscesses caused by the same pathogen, the enhanced immune response and the secretion of inflammatory factors led to an increased number of macrophage infiltration and thickening of the wall of the deep-brain abscess. Due to the small number of positive cases on bacterial culture, only the corresponding results of brain abscess mainly caused by Staphylococcus are available in the present study. Whether the wall thickness of brain abscesses caused by any other pathogenic microorganisms is positively correlated with the extent of macrophage infiltration requires to be further verified in larger studies.

However, in the present study, no difference in the thickness of the brain-abscess wall was obtained among the different types of pathogens. This result may be influenced by immune differences mainly caused by the various pathogenic microorganisms.

In the present study, 68 patients were successfully followed up, including 16 patients with deep-brain abscess. A total of 
10 patients (including 3 patients with abscesses in the deep brain) died after the surgery; all of these had a certain type of predisposing condition, such as history of operations, congenital heart disease, malignant tumors, cerebral hemorrhage or diabetes. Among the 10 patients, two patients died from malignant tumors and the cause of death in the remainder of the patients was associated with illnesses prior to treatment. During follow-up, there was no evidence of recurrence of brain abscess after treatment in either the living patients nor the deceased ones. Therefore, no statistical calculation or discussion of progression-free survival was performed. There was no statistically significant difference in the OS of patients with deep-brain abscess compared to those with superficial-brain abscess, which may be related to the less patients with superficial-brain abscess. Furthermore, there was no significant difference in OS among the different treatment groups. Therefore, in the present study, brain abscess had a low mortality rate $(12.00 \%)$, in comparison to the proportion of $40 \%$ in 1960 and $15 \%$ in the past decade (10). Of note, the mortality of the patients with deep-brain abscess (19\%) was higher than that of the patients with abscess in superficial brain regions (10\%), however, no significant difference was observed. However, the missing follow-up of certain patients may have influenced the accuracy of the outcomes reported in the present study.

In conclusion, the present study reported on the increased thickness of abscesses and the increased number of macrophages/microglia in the wall of deep-brain abscesses in comparison to shallow-brain abscesses. Brain abscesses at different sites caused by the same pathogen exhibited differences in wall thicknesses and extent of macrophage infiltration, suggesting that the thickness of the wall of brain abscesses in the deep brain may be associated with the extent of macrophage infiltration. Further studies are required to illustrate the formation mechanisms of abscesses in the deep and superficial brain. In addition, due to the high mortality and disability rate of deep brain abscesses and the poor effect of antibiotic treatment alone based on the previous treatment experiences, surgical treatment of abscesses combined with drug treatment is encouraged. Choosing the correct treatment method according to the pathological stage of an abscess and its location may improve patient outcomes.

\section{Acknowledgements}

Not applicable.

\section{Funding}

No funding was received.

\section{Availability of data and materials}

The datasets used and/or analyzed during the current study are available from the corresponding author on reasonable request.

\section{Authors' contributions}

SH designed the study. ZY, YY, NL and PW acquired important data and revised the manuscript for important intellectual content. YY, NL, ZY and XQ performed the histological examination of the samples. PW, ZY, LZ and MH analyzed the data. $\mathrm{ZY}$ and $\mathrm{SH}$ wrote the article. $\mathrm{ZY}$ and $\mathrm{SH}$ confirm the authenticity of all the raw data. All authors have read and approved the final manuscript.

\section{Ethics approval and consent to participate}

This study was approved by the Ethics Committee of Sanbo Brain Hospital (Beijing, China). Written informed consent for publication was obtained from all parents, legal guardians and participants.

\section{Patient consent for publication}

Not applicable.

\section{Competing interests}

The authors declare that they have no competing interests.

\section{References}

1. Carpenter J, Stapleton S and Holliman R: Retrospective analysis of 49 cases of brain abscess and review of the literature. Eur J Clin Microbiol Infect Dis 26: 1-11, 2007.

2. Reddy JS, Mishra AM, Behari S, Husain M, Gupta V, Rastogi M and Gupta RK: The role of diffusion-weighted imaging in the differential diagnosis of intracranial cystic mass lesions: A report of 147 lesions. Surg Neurol 66: 246-250-251, 2006.

3. Helweg-Larsen J, Astradsson A, Richhall H, Erdal J, Laursen A and Brennum J: Pyogenic brain abscess, a 15 year survey. BMC Infect Dis 12: 332, 2012.

4. Nicolosi A, Hauser WA, Musicco M and Kurland LT: Incidence and prognosis of brain abscess in a defined population: Olmsted County, Minnesota, 1935-1981. Neuroepidemiology 10: 122-131, 1991.

5. Felsenstein S, Williams B, Shingadia D, Coxon L, Riordan A, Demetriades AK, Chandler CL, Bassi S, Koutoumanou E, Stapleton S, et al: Clinical and microbiologic features guiding treatment recommendations for brain abscesses in children. Pediatr Infect Dis J 32: 129-135, 2013.

6. Nathoo N, Nadvi SS, Narotam PK and van Dellen JR: Brain abscess: Management and outcome analysis of a computed tomography era experience with 973 patients. World Neurosurg 75: 716-726; discussion 612-617, 2011.

7. Xiao F, Tseng MY, Teng LJ, Tseng HM and Tsai JC: Brain abscess: Clinical experience and analysis of prognosticfactors. Surg Neurol 63: 442-450, 2005.

8. Seydoux C and Francioli P: Bacterial brain abscesses: Factors influencing mortality and sequelae. Clin Infect Dis 15: 394-401, 1992.

9. Kao PT, Tseng HK, Liu CP, Su SC and Lee CM: Brain abscess: Clinical analysis of 53 cases. J Microbiol Immunol Infect 36: 129-136, 2003.

10. Brouwer MC, Coutinho JM and van de Beek D: Clinical characteristics and outcome of brain abscess: Systematic review and meta-analysis. Neurology 82: 806-813, 2014.

11. Brouwer MC, Tunkel AR, McKhann GM II and van de Beek D: Brain abscess. N Engl J Med 371: 447-456, 2014.

12. Tunkel AR: Brain abscess. In: Bennett JE, Dolin R and Blaser M, (eds). Principles and practice of infectious diseases. 8th edition. Philadelphia: Elsevier; pp1265-1272, 2015.

13. Britt RH, Enzmann DR and Yeager AS: Neuropathological and computerized tomographic findings in experimental brain abscess. J Neurosurg 55: 590-603, 1981.

14. Mathisen GE and Johnson JP: Brain abscess. Clin Infect Dis 25: 763-781, 1997.

15. Sauermann R, Karch R, Langenberger H, Kettenbach J, Mayer-Helm B, Petsch M, Wagner C, Sautner T, Gattringer R, Karanikas G and Joukhadar C: Antibiotic abscess penetration: Fosfomycin levels measured in pus and simulated concentration-time profiles. Antimicrob Agents Chemother 49: 4448-4454, 2005.

16. Gerding DN, Kozak AJ, Peterson LR and Hall WH: Failure of single doses of cefazolin and cefamandole to penetrate experimental chronic Escherichia coli abdominal abscesses. Antimicrob Agents Chemother 17: 1023-1029, 1980. 
17. Mutsaers SE, Bishop JE, McGrouther G and Laurent GJ: Mechanisms of tissue repair: From wound healing to fibrosis. Int J Biochem Cell Biol 29: 5-17, 1997.

18. Chambers RC, Laurent GJ and Westergren-Thorsson G: Cadmium inhibits proteoglycan and procollagen production by cultured human lung fibroblasts. Am J Respir Cell Mol Biol 19: 498-506, 1998

19. Song E, Ouyang N, Hörbelt M, Antus B, Wang M and Exton MS: Influence of alternatively and classically activated macrophages on fibrogenic activities of human fibroblasts. Cell Immunol 204: 19-28, 2000.

20. Aldrich A and Kielian T: Central nervous system fibrosis is associated with fibrocyte-like infiltrates. Am J Pathol 179: 2952-2962, 2011

21. Villa C, Miquel C, Mosses D, Bernier M and Di Stefano AL: The 2016 World Health Organization classification of tumours of the central nervous system. Presse Med 47: e187-e200, 2018.

22. Han S, Wang PF, Xing YX, Song HW, Yao K and Lin ZX: Human Cytomegalovirus (HCMV) infection was not correlated with overall survival in glioblastomas. Neoplasma 65: 431-435, 2018.

23. Gould PV and Saikali S: A comparison of digitized frozen section and smear preparations for intraoperative neurotelepathology. Anal Cell Pathol (Amst) 35: 85-91, 2012.

24. Zheng PP, van der Weiden M and Kros JM: Fast tracking of co-localization of multiple markers by using the nanozoomer slide scanner and NDPViewer. J Cell Physiol 229: 967-973, 2014

25. Meneghin A and Hogaboam CM: Infectious disease, the innate immune response, and fibrosis. J Clin Invest 117: 530-538, 2007.

26. Bhatia R, Tandon P and Misra NK: Inflammatory lesions of the basal ganglia and thalamus: Review of twenty-one cases. Neurosurgery 19: 983-988, 1986.

27. Burt AD: Pathobiology of hepatic stellate cells. J Gastroenterol 34 299-304, 1999.

28. Phan SH: Role of the myofibroblast in pulmonary fibrosis. Kidney Int Suppl 54 (Suppl): S46-S48, 1996.

29. Clouthier DE, Comerford SA and Hammer RE: Hepatic fibrosis, glomerulosclerosis, and a lipodystrophy-like syndrome in PEPCK-TGF-beta1 transgenic mice. J Clin Invest 100: 2697-713, 1997.

30. Kielian T and Hickey WF: Proinflammatory cytokine, chemokine, and cellular adhesion molecule expression during the acute phase of experimental brain abscess development. Am J Pathol 157: 647-658, 2000.

31. Baldwin AC and Kielian T: Persistent immune activation associated with a mouse model of Staphylococcus aureus-induced experimental brain abscess. J Neuroimmunol 151: 24-32, 2004.

32. Kielian T: Immunopathogenesis of brain abscess J Neuroinflammation 1: 16, 2004.

33. Lawson LJ, Perry VH, Dri P and Gordon S: Heterogeneity in the distribution and morphology of microglia in the normal adult mouse brain. Neuroscience 39: 151-710, 1990.

34. Enzmann DR, Britt RH and Yeager AS: Experimental brain abscess evolution: Computed tomographic and neuropathologic correlation. Radiology 133: 113-122, 1979.

35. Obana WG, Britt RH, Placone RC, Stuart JS and Enzmann DR: Experimental brain abscess development in the chronically immunosuppressed host. Computerized tomographic and neuropathological correlations. J Neurosurg 65: 382-391, 1986.

36. Kielian T, Barry B and Hickey WF: CXC chemokine receptor-2 ligands are required for neutrophil-mediated host defense in experimental brain abscesses. J Immunol 166: 4634-4643, 2001.

37. Kielian T, Cheung A and Hickey WF: Diminished virulence of an alpha-toxin mutant of Staphylococcus aureus in experimental brain abscesses. Infect Immun 69: 6902-6911, 2001.

38. Colton CA: Heterogeneity of microglial activation in the innate immune response in the brain. J Neuroimmune Pharmacol 4 399-418, 2009
39. van Rossum D, Hilbert S, Strassenburg S, Hanisch UK and Brück W: Myelin-phagocytosing macrophages in isolated sciatic and optic nerves reveal a unique reactive phenotype. Glia 56: 271-283, 2008

40. Herold R, Schroten $\mathrm{H}$ and Schwerk C: Virulence factors of meningitis-causing bacteria: Enabling brain entry across the blood-brain barrier. Int J Mol Sci 20: 5393, 2019.

41. Dando SJ, Mackay-Sim A, Norton R, Currie BJ, St John JA, Ekberg JA, Batzloff M, Ulett GC and Beacham IR: Pathogens penetrating the central nervous system: Infection pathways and the cellular and molecular mechanisms of invasion. Clin Microbiol Rev 27: 691-726, 2014.

42. Iovino F, Orihuela CJ, Moorlag HE, Molema G and Bijlsma JJ: Interactions between blood-borne Streptococcus pneumoniae and the blood-brain barrier preceding meningitis. PLoS One 8: e68408, 2013.

43. Shainheit MG, Mulé M and Camilli A: The core promoter of the capsule operon of Streptococcus pneumoniae is necessary for colonization and invasive disease. Infect Immun 82: 694-705, 2014.

44. Gratz N, Loh LN, Mann B, Gao G, Carter R, Rosch J and Tuomanen EI: Pneumococcal neuraminidase activates TGF- $\beta$ signalling. Microbiology 163: 1198-1207, 2017.

45. Kim KS: Human meningitis-associated Escherichia coli. EcoSal Plus: May 1, 2016 (Epub ahead of print). doi: 10.1128/ecosalplus. ESP-0015-2015.

46. Khan NA, Shin S, Chung JW, Kim KJ, Elliott S, Wang Y and Kim KS: Outer membrane protein A and cytotoxic necrotizing factor-1 use diverse signaling mechanisms for Escherichia coli $\mathrm{K} 1$ invasion of human brain microvascular endothelial cells. Microb Pathog 35: 35-42, 2003.

47. Huang SH, Wan ZS, Chen YH, Jong AY and Kim KS: Further characterization of Escherichia coli brain microvascular endothelial cell invasion gene ibeA by deletion, complementation, and protein expression. J Infect Dis 183: 1071-1078, 2001.

48. Wang $\mathrm{MH}$ and Kim KS: Cytotoxic necrotizing factor 1 contributes to Escherichia coli meningitis. Toxins (Basel) 5: 2270-2280, 2013.

49. Koutsouras GW, Ramos RL and Martinez LR: Role of microglia in fungal infections of the central nervous system. Virulence 8: $705-718,2017$.

50. van de Veerdonk FL, Kullberg BJ, van der Meer JW, Gow NA and Netea MG: Host-microbe interactions: Innate pattern recognition of fungal pathogens. Curr Opin Microbiol 11: 305-312, 2008.

51. Yang KY, Chang WN, Ho JT, Wang $\mathrm{HC}$ and Lu CH: Postneurosurgical nosocomial bacterial brain abscess in adults. Infection 34: 247-251, 2006.

52. Kielian T, Phulwani NK, Esen N, Syed MM, Haney AC, McCastlain K and Johnson J: MyD88-dependent signals are essential for the host immune response in experimental brain abscess. J Immunol 178: 4528-4537, 2007.

53. Kielian T, Mayes P and Kielian M: Characterization of microglial responses to Staphylococcus aureus: Effects on cytokine, costimulatory molecule, and Toll-like receptor expression. J Neuroimmunol 130: 86-99, 2002.

54. Kielian T, Esen N, Bearden ED: Toll-like receptor 2 (TLR2) is pivotal for recognition of $S$. aureus peptidoglycan but not intact bacteria by microglia. Glia 49: 567-756, 2005.

(i) $\Theta$ This work is licensed under a Creative Commons Attribution-NonCommercial-NoDerivatives 4.0 International (CC BY-NC-ND 4.0) License. 